\title{
Three dimensional tactile-optical probing for the measurement of microparts
}

\author{
Rainer Tutsch ${ }^{1}$, Matthias Andräs ${ }^{2}$, Ulrich Neuschaefer-Rube ${ }^{3}$, Marcus Petz ${ }^{1}$, \\ Thomas Wiedenhöfer ${ }^{2}$, Mark Wissmann ${ }^{3}$ \\ 1) Institut für Produktionsmesstechnik, TU Braunschweig, Schleinitzstraße 20, 38106 Braunschweig \\ 2) Werth Messtechnik GmbH, Siemensstraße 19, 35394 Gießen \\ 3) Physikalisch-Technische Bundesanstalt, Bundesallee 100, 38116 Braunschweig
}

\begin{abstract}
The determination of structures on microparts requires particularly sensitive measuring devices. Here the so called fiber probe is an outstanding, established solution. As probing forces are in the order of some $\mu \mathrm{N}$ the surface of the object under test will not be damaged by the probing element. Furthermore effects of the probing force on the measurement uncertainty can be neglected. Beyond that so far inaccessible micro geometries (like e.g. deep, small holes with diameters less than $50 \mu \mathrm{m}$ ) can be measured easily. The tactile-optical method of measurement based on this principle does not include full 3D-capabilities so far. New developments to overcome this limitation are the stereoscopic detection of the probing sphere and the speckle pattern based analysis of the $z$-deflection of the probing element.
\end{abstract}

\section{Tactile-optical metrology for the measurement of microparts}

Tactile-optical measurements are carried out by use of so called fiber-probes. The probing element - a ball-tip with a diameter of ten to some hundred micrometers - is located at the end of a flexible bended glass fiber situated in the focal plane of an optical CMM. The measurement can be carried out by use of backlight illumination - measuring the resulting shadow of the ball-tip - or by detecting the position of the illuminated probing sphere (LED illumination through the shaft) by use of the optical CMM (Fig. 1). Aside very small diameters of the probing element the very small probing forces $(1 \mu \mathrm{N}$ to $100 \mu \mathrm{N})$ predestine this principle of measurement for the measurement of sensitive and small parts. The xy-deflection of the probing element is detected by the camera system and the position of the probing points is calculated by image processing.
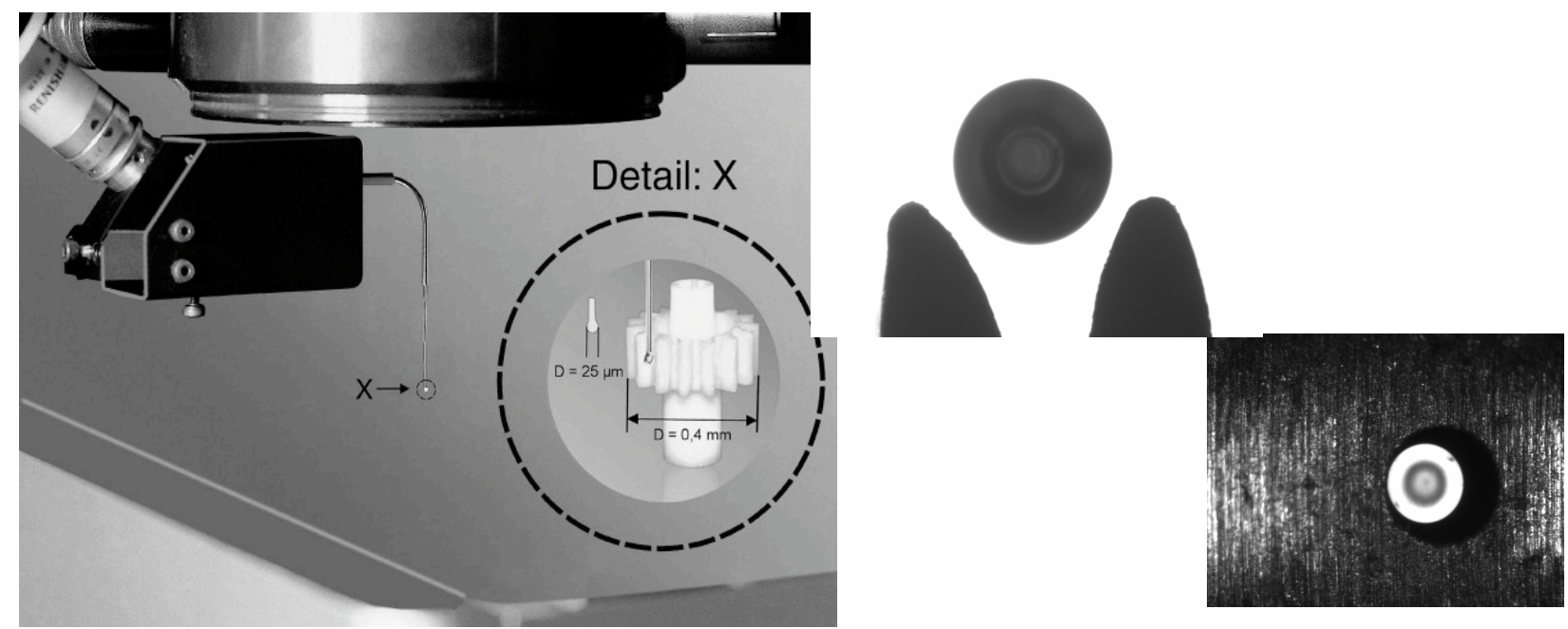

Fig. 1 Tactile-optical probing of microparts by use of a fiber-probe

The extension of the measurement system by a second sphere located on the probing shaft above the probing element eliminates measurement deviations resulting from the penetration depth (e.g. in small deep holes). The position of the additional sphere is detected and the probing point is calculated. A further optimization of the measurement method is the use of an piezo translator which induces a permanent oscillation of the fiber probe in z-direction (patended). Fig. 2 shows the difference in the 
characteristic path of the probing sphere with (Fig.2 right) and without (Fig. 2 left) fiber oscillation. The oscillation ensures that friction effects (e.g. stick slip) are reduced. Additionally an appropriate coating reduces the coefficient of friction.
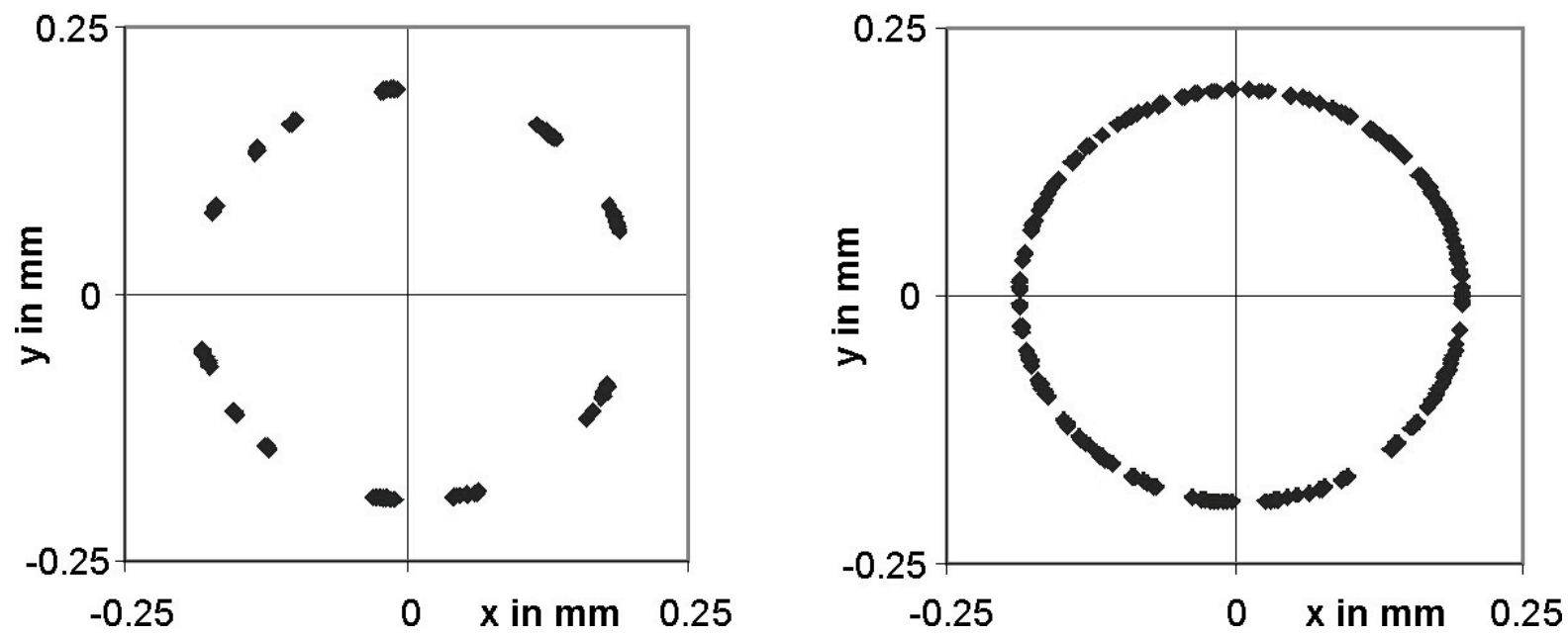

Fig. 2 Scanning without (left) with (right) fiber oscillation

\section{Advantages of fiber probe:}

- smallest 3D features can be measured (probe spheres from $10 \mu \mathrm{m}$ up to $500 \mu \mathrm{m}$ )

- negligible probing force ("nearly non-contact")

- scanning operation

- most accurate probe worldwide

- no influence of stylus length and probe electronics on the results

- calibration of optical measurements

- roughness measurements

Typical microparts to be measured are for example micro gears for gear boxes which are used in small pumps (e.g. for insulin distribution, dental-drills, etc.) (Fig. 3).
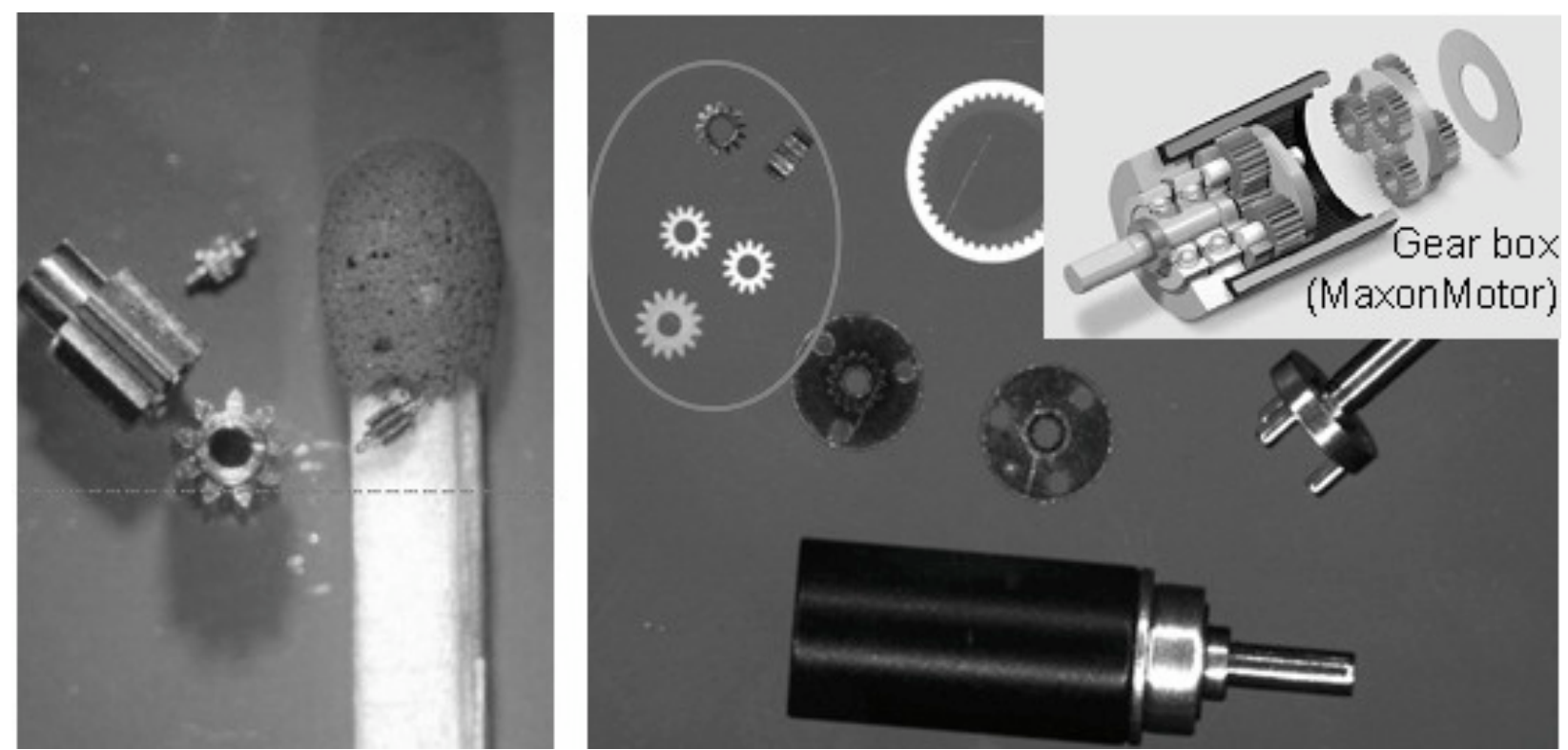

Fig. 3 dimensions of typical microparts - gears and gear box [1]

In Fig. 4 a typical measurement result is represented. The gear under test can be measured along the surface lines in different depths. Even the small center-hole can be measured. The information about the third dimension (z-direction) is provided by the scales of the CMM. 
A limitation of the fiber probing described above is, that it can not be used to detect deflections in $z$ as well. As the depth can not be determined directly (only by observing the behavior of the fiber when moving along perpendicular side walls - on the top a "snap-out" effect can be observed) with adequate accuracy new approaches have been developed. Two different approaches are described in chapter 2 .
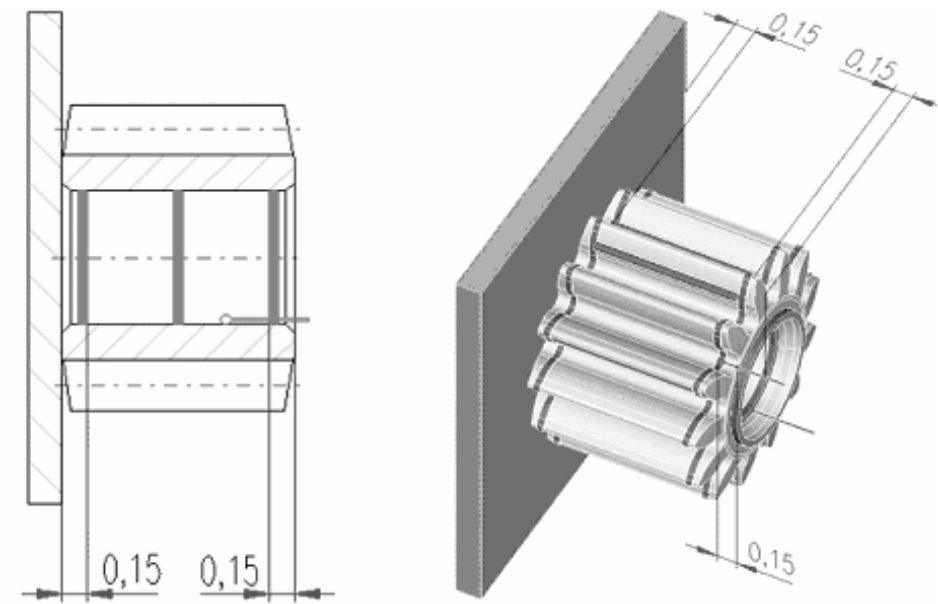

Fig. 4 Measurement of a microgear using a fiber probe [1]

\section{Advances in three dimensional fiber probing}

\subsection{Stereoscopic fiber-probing}

Photogrammetry is a well established method for determining the three-dimensional position of an object from a series of images taken from different directions. In stereo-photogrammetric setups usually two separate cameras are used simultaneously to obtain two different views of the object under test. By installing a special mirror optics setup in front of a conventional camera lens, however, it is possible to simultaneously generate two different views of an object on the image sensor of one single camera. An according mirror optic was designed to be magnetically mounted to the Werth IP40 camera head (Fig. 5).
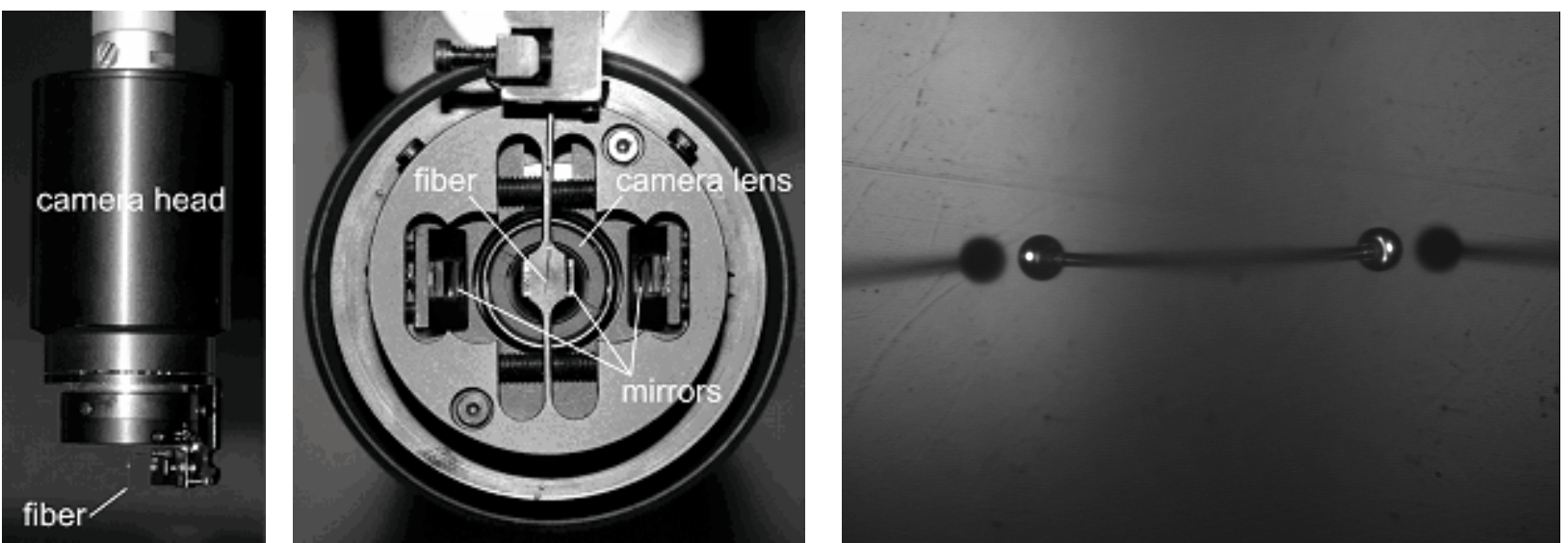

Fig. 5 Stereoscopic sensor head based on Werth IP 40 (left: overall view, middle: bottom view, right: single CCD image showing two different views of the probing element)

As illustrated in Fig. 5, right the mirror setup splits the camera's field of view in half, thus allowing to simultaneously observe the probing element at the end of the fiber from two different directions. From this image data the three-dimensional position of the probing element can be determined by photogrammetric methods. Since the IP 40's camera objective is telecentric the photogrammetric camera model has been extended to support both central and parallel projection.

The characteristics of the optical system are:

- optical magnification approx. 4

- standard CCD-camera $760 \times$ of 570 pixels 
- working distance approx. $20 \mathrm{~mm}$

- triangulation angle $35^{\circ}$

The determination of the probing error $\mathrm{P}$ has been carried out according to EN ISO 10360-2: 2001. The distribution of the 25 points to be probed had to be varied deviant from the recommended strategy since some recommended points were not detectable due to the design of the probe (only one spherical probing element). The adapted distribution is illustrated in Fig. 6.

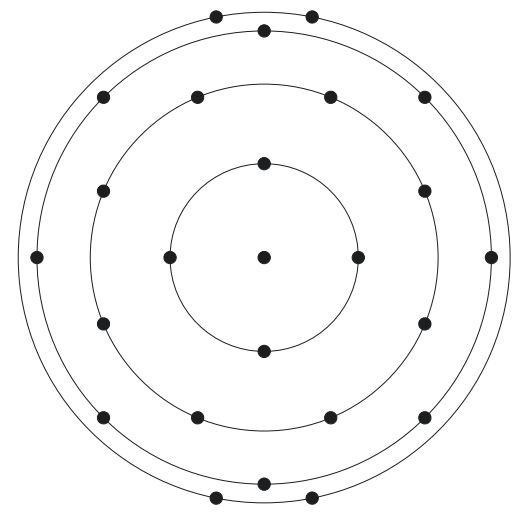

Fig. 6 Point distribution for the probing error calculation

The measurement of these 25 points (Fig. 6) has been repeated 125 times by use of a calibrated sphere. The probing deflection of the fiber probe has been varied in 25 steps. Fig. 7 illustrates the minimum, maximum and average value of the radial deviations from the best-fit sphere for each of the 25 points. The maximum range of the radial distances (means probing error P; over 125 runs determined instead of a single measurement) has been calculated as $2.165 \mu \mathrm{m}$. The range of the determined radii of the best-fit sphere has been determined as $0.5 \mu \mathrm{m}$.

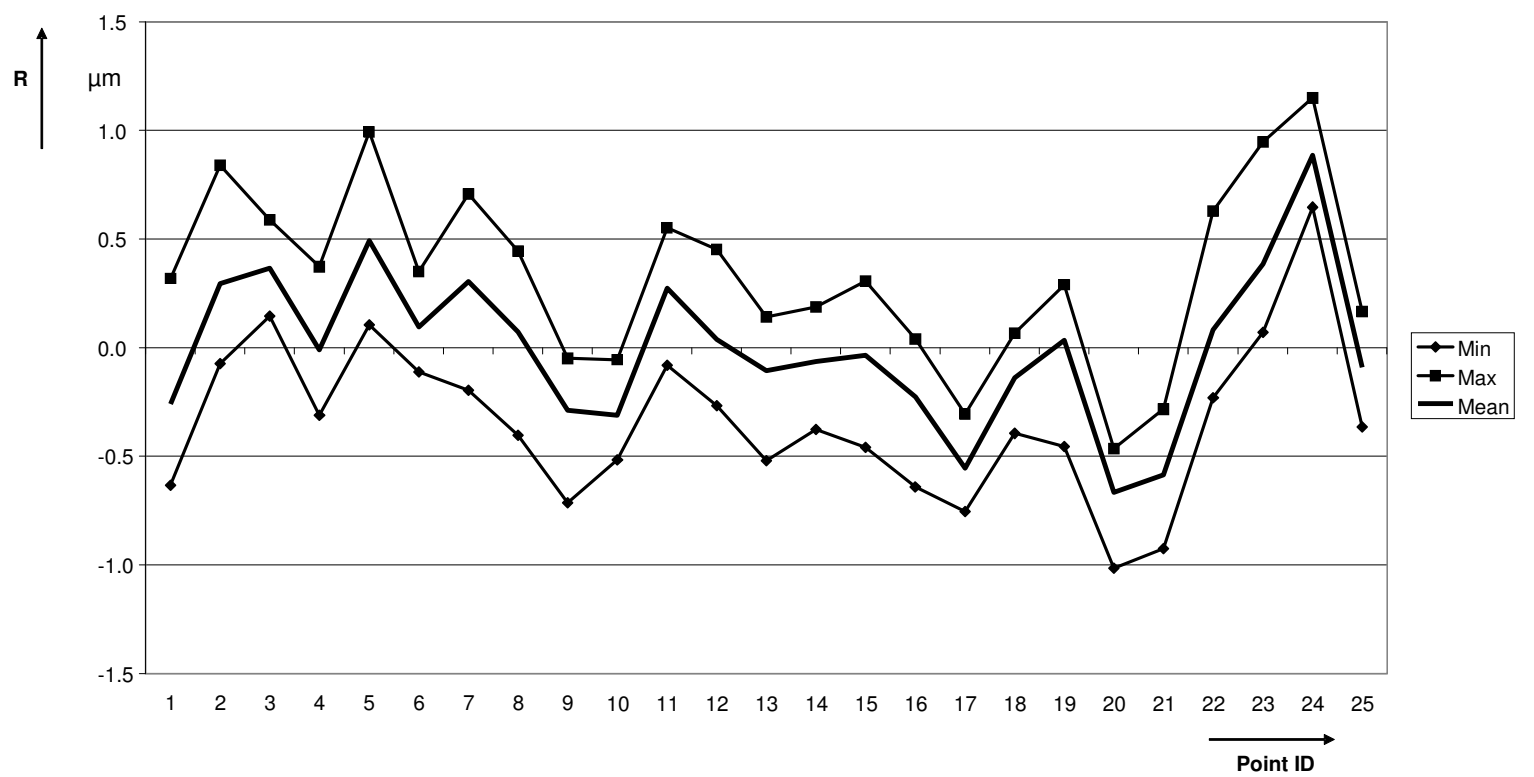

Fig. 7 Radial deviations for the measurement of a calibrated sphere with the stereoscopic measurement setup

Temperature effects to the IP 40 camera head have been identified as a main source of statistical errors. These effects could be reduced in the meantime by a redesign of the camera head. The systematic errors are most likely caused by the form deviation of the probing sphere. Here calibration and correction of the probing sphere diameter can be carried out. Fig. 8 shows the same results as Fig. 7, but with a correction of the systematic errors. The maximum range of the radial deviations is reduced to $1.078 \mu \mathrm{m}$. 


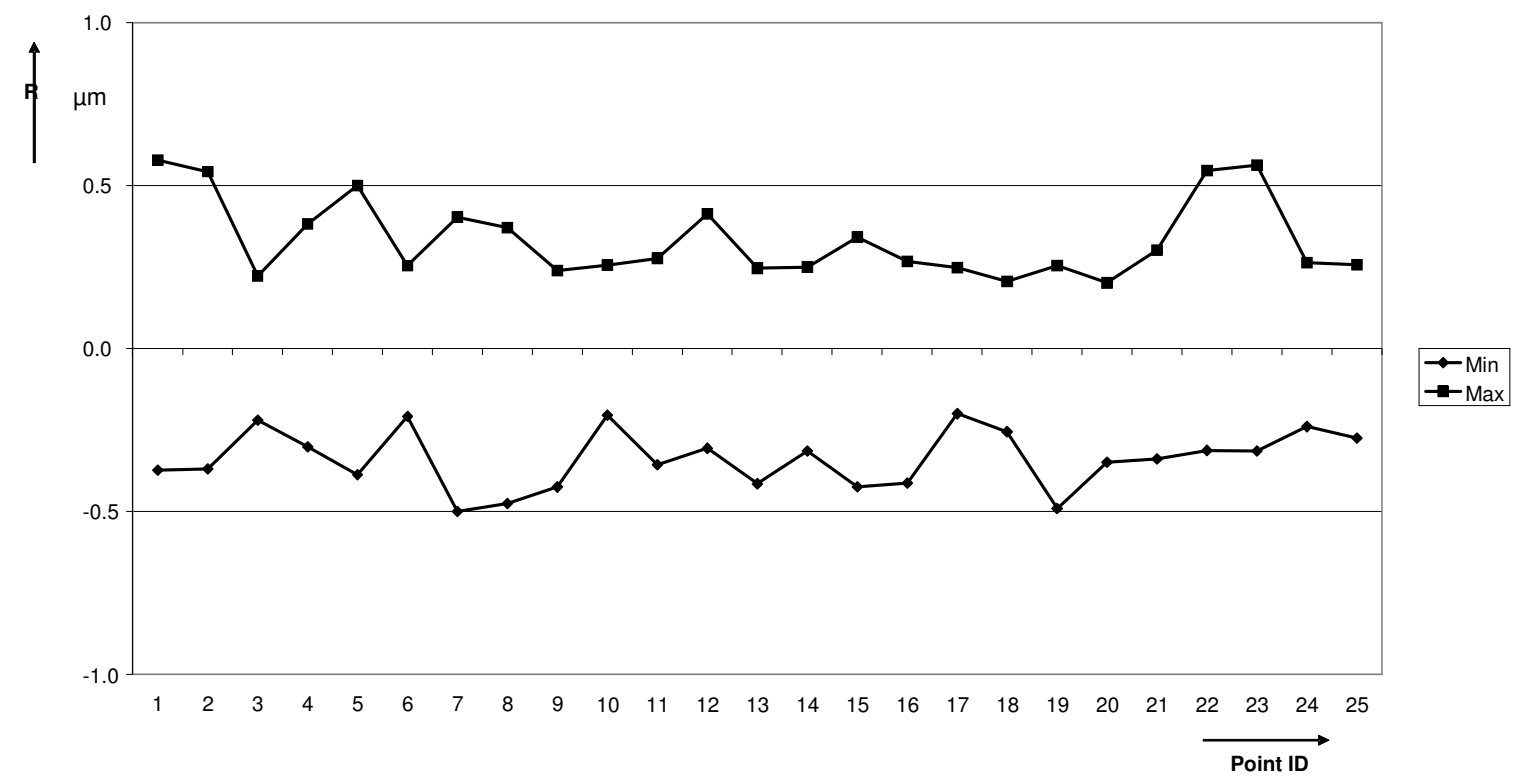

Fig. 8 Corrected radial deviations for the measurement of a calibrated sphere with the stereoscopic setup

\subsection{Fiber probing based on an speckle pattern analysis}

In this setup the LED used in the standard fiber probe setup is replaced by a multimode laser diode emitting coherent light. In addition, a fiber probe with reduced z-stiffness (Fig. 9, left) and a second, sphere with diffuse reflective coating at the probing shaft (Fig. 9, middle) is used. Due to the coherent illumination, a speckle pattern (Fig. 9, right) is detected on the upper sphere. The speckle pattern is caused by different optical path lengths in the fiber and is therefore stable in relation to the second sphere. This is utilized to localize the second sphere in the $x$ - und $y$-direction by correlation techniques The speckle texture is dependent on the z-deflection (Fig. 10). The z-deflection is determined by locating the measured speckle pattern in a reference stack consisting of reference speckle pattern images with known z-deflections by correlation techniques (Fig. 11). The z-position can be determined with submicron accuracy $(<0,5 \mu \mathrm{m}$, peak to valley).
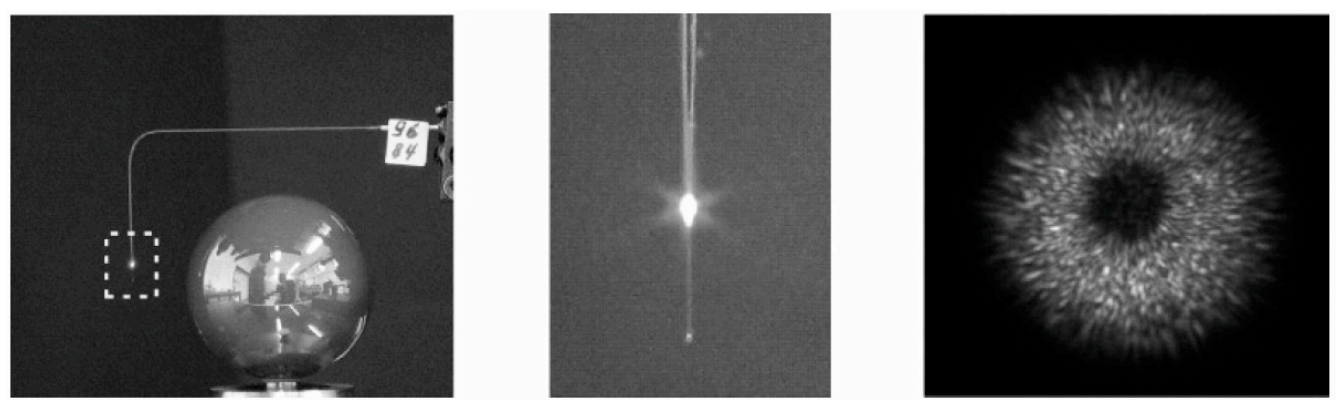

Fig. 9 Set-up of the speckle-based analysis with calibrated sphere $(\varnothing 25 \mathrm{~mm})$ (left); detail of the illuminated upper probing sphere (middle); speckle pattern observed by the CCD-Camera of the CMM [2]
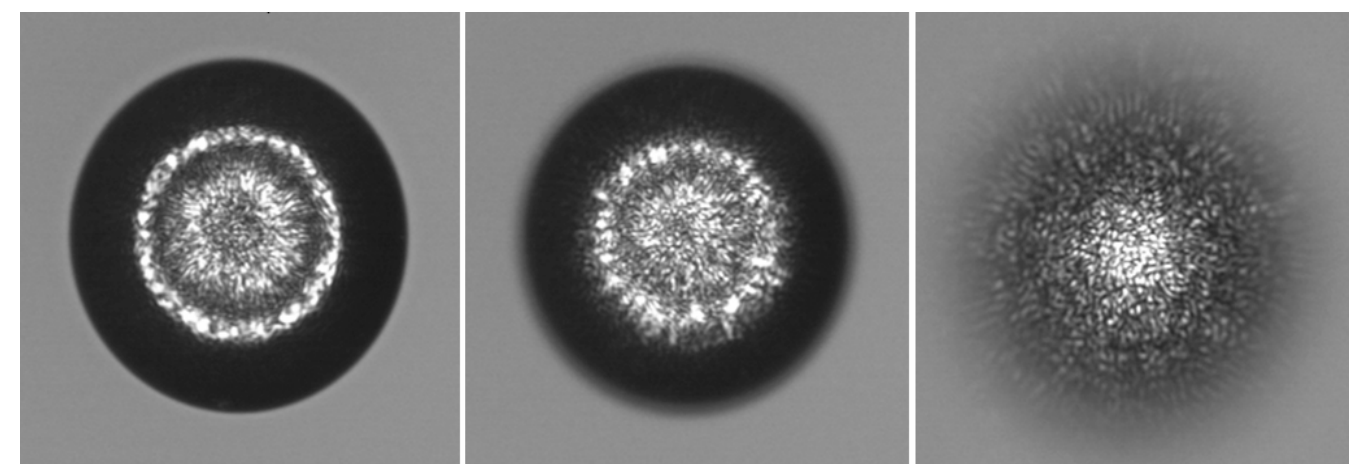

Fig. 10 Speckle pattern for different $z$-deflections. From left to right: $d z=0 \mu \mathrm{m} ; \mathrm{dz}=50 \mu \mathrm{m}, \mathrm{dz}=300 \mu \mathrm{m}$ 

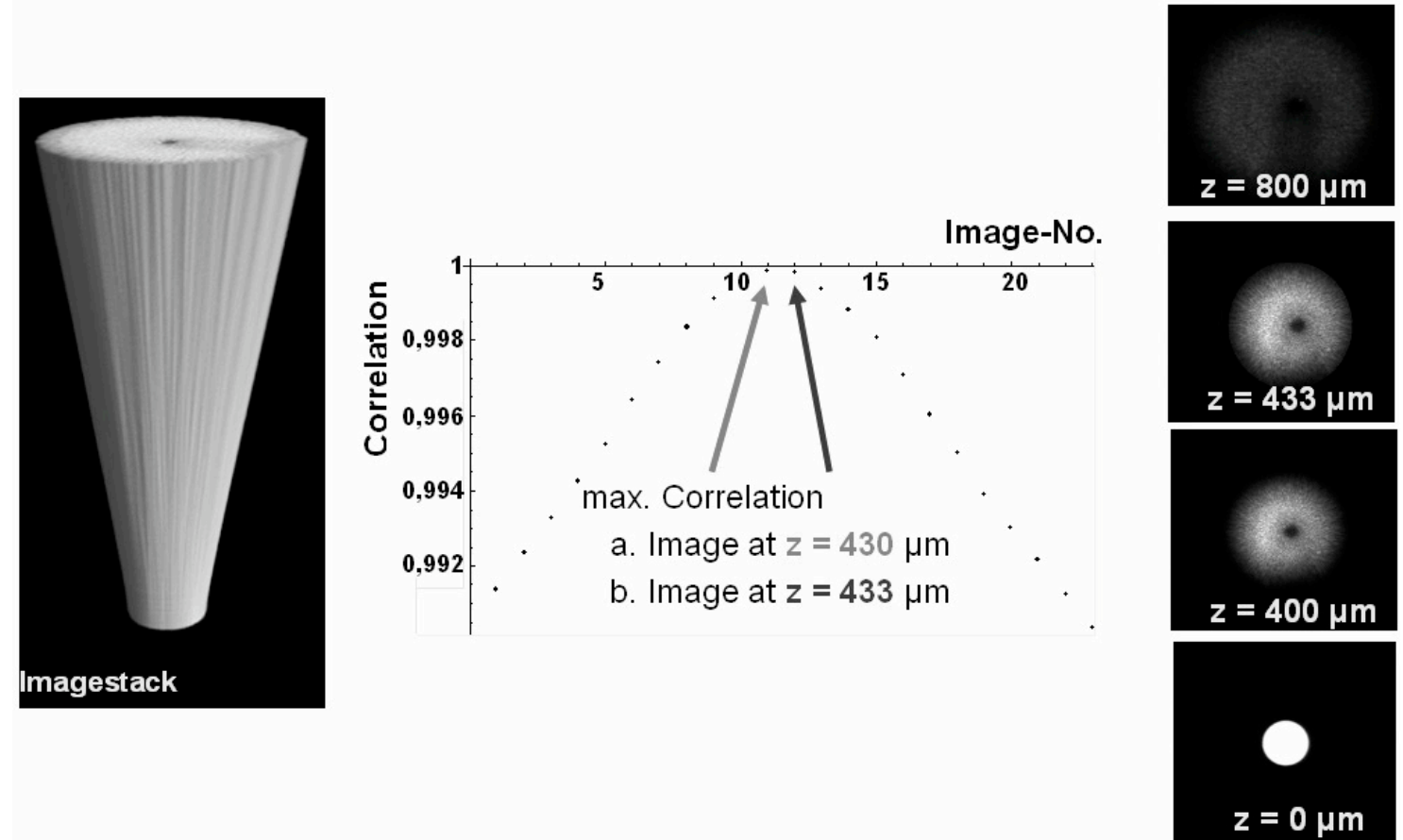

Fig. 11 Determination of z-position by calculating the correlation of detected speckle pattern by use of a reference stack consisting of reference speckle pattern images with known z-deflections.

The accuracy of the speckle correlation method has been evaluated (similar to the procedure described for the stereoscopic setup) by measurement of an calibrated sphere according to EN ISO 10360-2: 2001. Fig. 12 shows the result of the repeated measurement of a calibrated sphere $(\varnothing 25 \mathrm{~mm}$, negligible form deviation). The maximum deviation of the 468 measured points of the best-fit sphere is $0.8 \mu \mathrm{m}$.

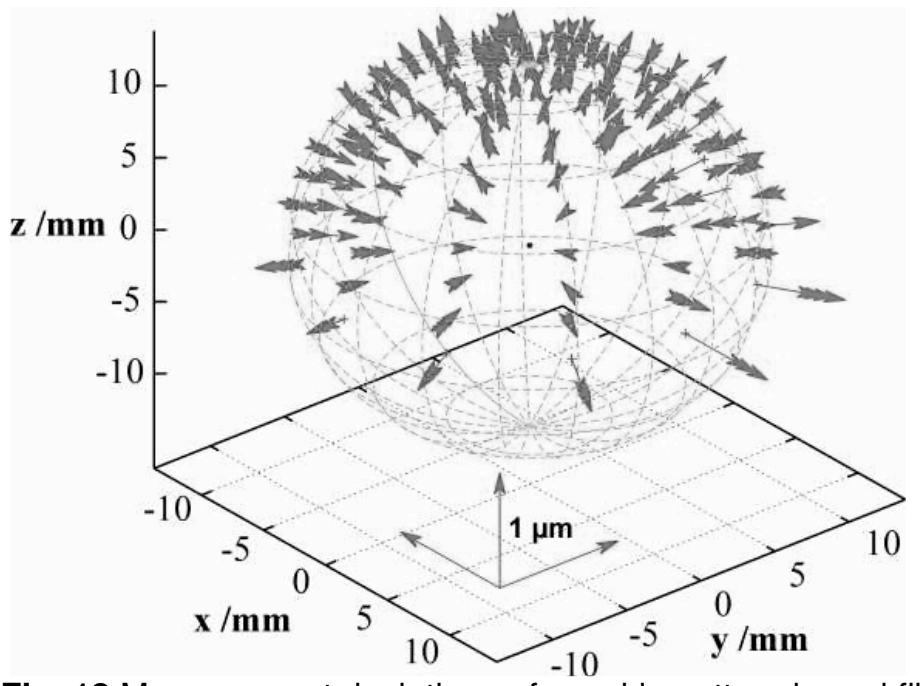

Fig. 12 Measurement deviations of speckle pattern based fiber probing

The image processing algorithms for the evaluation of the speckle pattern were accelerated in such a manner that now up to 20 measurements per second (with standard computer hardware) are possible. The probe is therefore also applicable for measurements in scanning mode.

\section{References}

[1] BMBF-funded research project MODUS (supporting programme "Mikrosysteme 2004-2009").

[2] U. Neuschaefer-Rube, M. Wissmann; "Tactile-optical 3D sensor applying image processing"; Proc. SPIE, Vol. 7239, 72390H1 - 72390H9 (2009); DOI:10.1117/12.810464.

[3] R. Christoph, H.-J. Neumann: "Multisensorkoordinatenmesstechnik"; Bibliothek der Technik Band 248. 